\title{
Neoadjuvant PRRT for advanced pNEN: an unusual highlander
}

\author{
Isabella Zanata ${ }^{1} \cdot$ Maria Rosaria Ambrosio $^{1} \cdot$ Maria Chiara Zatelli ${ }^{1}{ }^{1}$
}

Received: 14 January 2021 / Accepted: 10 February 2021 / Published online: 4 March 2021

(c) The Author(s) 2021

Neoadjuvant treatment may be useful in inoperable pancreatic neuroendocrine neoplasms (pNEN). A 41-year-old man complained for severe gastrointestinal symptoms (severe dysphagia, gastroesophageal reflux, postprandial diarrhoea, and weight loss); abdominal ultrasound showed two liver nodules and computerized tomography (CT) showed multiple liver metastases and a $4 \mathrm{~cm}$ highly vasularized pancreatic mass, suspected for NEN (Fig. 1a, b). Chromogranin A $(\mathrm{CgA})$, Neuron Specific Enolase, gastrin, glucose and insulin levels, blood count, and liver, kidney, adrenal, and pituitary function were normal. Calcitonin levels were high (151 pg/ $\mathrm{ml})$. Somatostatin receptor (SSTR) scintigraphy (Octreoscan) showed a strong uptake in the pancreatic tumor and liver metastases (Fig. 1c); the patient started medical therapy with a long acting somatostatin analogue (SSA) in keeping with current guidelines [1] and with a strong Octreoscan positivity. Surgical exploration with multiple biopsies showed a locally invasive, well-differentiated G1 (Ki-67 index $<2 \%$ ) NEN with positive immunohistochemistry for calcitonin, $\mathrm{CgA}$ and SSTR subtypes $1,2,3$, and 5 ( $\mathrm{pNEN} \mathrm{cT}_{4} \mathrm{~N}_{\mathrm{X}} \mathrm{M}_{1 \mathrm{~A}}$; Stage IV), according to AJCC TNM classification (8th edition). Surgical management was contraindicated at that time due to portal and splenic vein involvement. A systemic neoadjuvant treatment was needed in order to manage the disease with the perspective of a future surgical treatment to allow tumor removal and prolong survival of this young patient. Everolimus and sunitinib are approved treatments after SSA failure, but were unavailable at that time. Cytotoxic chemotherapy is indicated as first-line therapy in G2 pNEN, which was not our case. Ablative loco-regional therapies could have addressed liver disease, leaving untreated the pancreatic mass [2]. Therefore, we opted for peptide receptor radionuclide therapy (PRRT), despite this approach is not routinely employed in

Maria Chiara Zatelli

ztlmch@unife.it

1 Section of Endocrinology and Internal Medicine, Department of Medical Sciences, University of Ferrara, Ferrara, Italy neoadjuvant and adjuvant settings in pNEN, in keeping with the inclusion criteria indicated by the ENETS 2009 Consensus Guidelines [3]. PRRT is recommended in nonfunctional G1-G2 unresectable pNEN after failure of medical therapy and it may improve symptoms and quality of life in patients with functioning pNEN [4]. Our patient underwent five PRRT cycles with ${ }^{90}$ Yttrium $\left({ }^{90} \mathrm{Y}\right)$-DOTATOC $(10.619 \mathrm{MBq}$ cumulative dose in 9 months), without side effects. The patient was treated with Y-DOTATOC because that was the only PRRT treatment available at the time the patient was addressed to this approach.

Post PRRT abdominal CT showed a $>50 \%$ reduction in pancreatic tumor size (maximum diameter from 4 to $1.3 \mathrm{~cm}$ ) and disappearance of liver metastases (Fig. 1d, e), but persistence of splenic vein occlusion, as confirmed by ${ }^{68}$ Gallium-DOTANOC PET-CT (Fig. 1f). Calcitonin levels $(2.7 \mathrm{pg} / \mathrm{ml})$, blood glucose and insulin levels were normal. The case was discussed in the NEN tumor board and the patient was found eligible for distal pancreatectomy and splenectomy. Histopathology showed a $2 \mathrm{~cm}$ welldifferentiated NEN with Ki-67 index $=1 \%$ (Grade 1) with perineural invasion ( $\mathrm{pNEN} \mathrm{pT}_{1} \mathrm{~N}_{0} \mathrm{M}_{0}$, Stage I). Post-surgical abdominal CT showed the absence of liver metastases, portal vein invasion and pancreatic recurrence (Fig. 1g, h), as confirmed by ${ }^{68}$ Gallium-DOTANOC PET-CT (Fig. 1i). Calcitonin levels were undetectable $(<1 \mathrm{pg} / \mathrm{ml})$. Long-term yearly follow-up ( $>9$ years) evaluated biochemistry, abdominal CT and ${ }^{68}$ Gallium-DOTATOC PET-CT and allowed to observe a progression-free survival (PFS) after neoadjuvant PRRT of $>108$ months, which is threefold to fourfold longer as compared to previously observed results. PRRT indeed caused a tumor down-staging from Stage IV to Stage I, allowing complete surgical resection. Eight years after PRRT and 7 years after surgery, SSA therapy was stopped. Two years after SSA treatment withdrawal, no biochemical nor morpho-functional evidence of recurrence has been detected and the patient is free of disease 11 years after initial diagnosis (Fig. 11-n).

Neoadjuvant PRRT has already been reported to reduce NEN bulk in cases that were not eligible for surgery at 

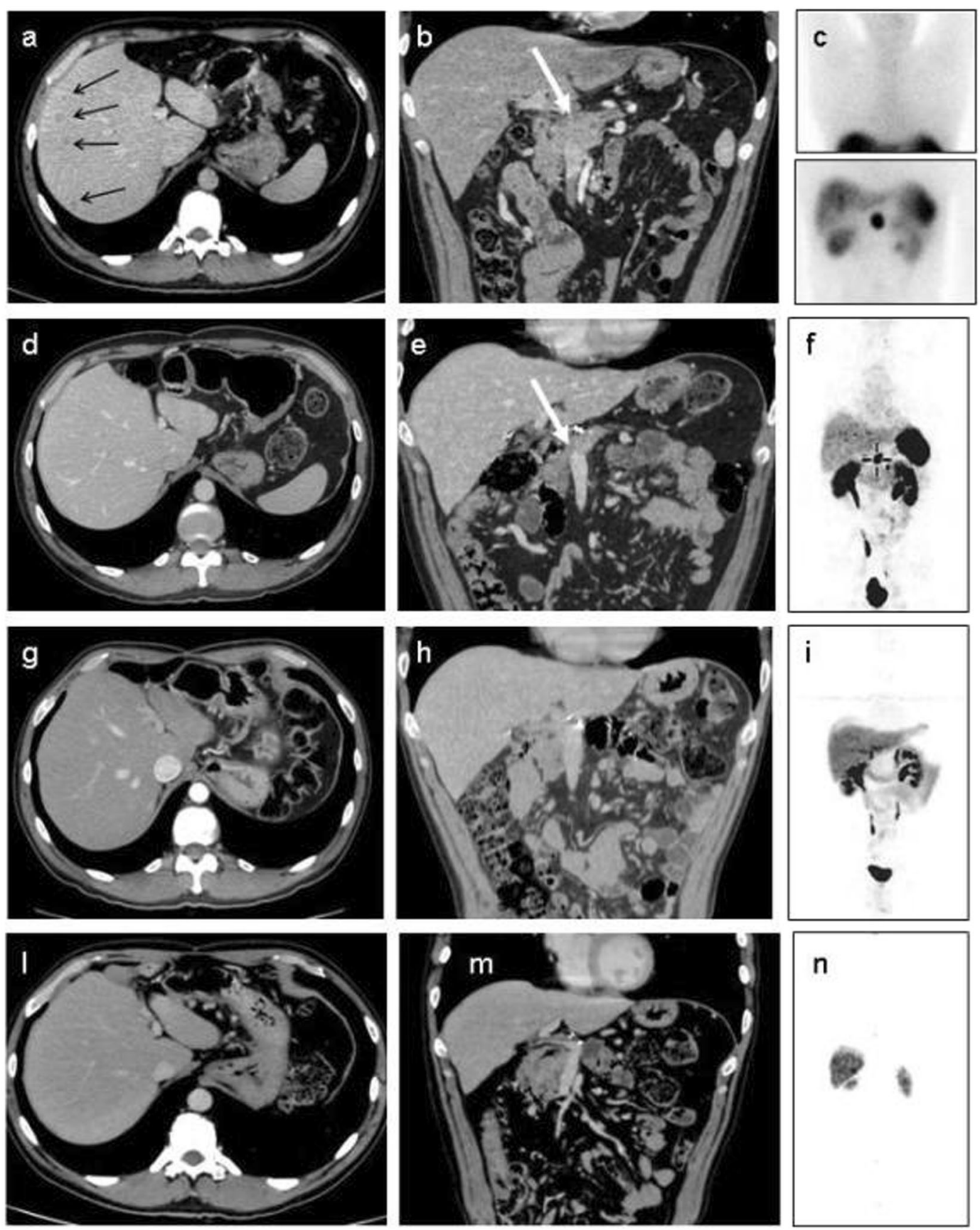

Fig. 1 Clinical case imaging. Imaging at the time of diagnosis. Axial portal venous phase CT (a) shows multiple metastases in the right liver lobe (arrows). Multiplanar coronal reconstruction (b) shows a $4 \mathrm{~cm}$ mass (white arrow) in the pancreatic body. Anterior planar Octreoscan scan (c) confirms the pathological intense tracer uptake in the pancreatic tumor and liver metastases. The patient provided written informed consent for disclosing clinical and imaging data. Follow-up imaging 5 months after PRRT. Axial portal venous phase CT scan (d) shows the absence of liver metastases and the reduction $(>50 \%)$ in the pancreatic mass (white arrow) (e). Anterior planar ${ }^{68}$ GalliumDOTANOC PET-CT scan (f) confirms the absence of the

diagnosis [5-10] also in pNEN. Recently, PRRT efficacy was demonstrated in pNEN in neoadjuvant settings, showing that patients previously treated with PRRT have a lower pathological uptake in the liver and a radiotracer uptake reduction in the pancreatic tumor. g-i Imaging 3 months after surgery. Axial CT scan (g) shows post-operative findings of distal pancreatectomy and splenectomy with a persistent absence of liver metastases. On coronal CT image (h), the pancreas head is free of local recurrence. Anterior planar ${ }^{68}$ Gallium-DOTANOC PET-CT scan (i) confirms the absence of the pathological uptake both in the liver and in the residual pancreas. Imaging $\sim 10$ years after surgery. Axial-coronal CT scans $(\mathbf{l}, \mathbf{m})$ and anterior planar ${ }^{68}$ Gallium-DOTANOC PET-CT scan (n) overlap with the previous morpho-functional imaging and show the absence of recurrence both in the liver and in the residual pancreas

incidence of nodal metastases at the time of surgery, reduced intra- and post-surgical complications and longer PFS (52 months) as compared to patients treated only with surgery 
(37 months) [11]. We found 6 studies describing a total of 21 patients undergoing neoadjuvant PRRT for an unoperable pNEN, who eventually underwent surgery obtaining a complete tumor resection ( $\mathrm{R} 0$ resection) [5, 12-16].

Our clinical experience is in line with current literature on pNEN and proves the potential efficacy of neoadjuvant 90YPRRT in G1 metastatic pNEN. PRRT, indeed, may improve life expectancy and long-term prognosis after surgery, without major side effects. Current guidelines do not provide specific indication concerning adjuvant SSA therapy duration after PRRT: our case underlines the importance of a multidisciplinary evaluation in order to tailor the treatment according to patient's characteristics.

Acknowledgements The Authors would like to thank their Colleagues for their precious support for this manuscript Fabio Pellegrino and Melchiore Giganti, of the Section of Radiology, Department of Morphology, Surgery and Experimental Medicine, University of Ferrara, Ferrara, Italy; Massimo Falconi of the Pancreatic Surgery Unit, Pancreas Translational \& Clinical Research Center, San Raffaele Hospital IRCCS, "Vita-Salute" University, Milan, Italy; Mirco Bartolomei of the Nuclear Medicine Department, "S. Anna" Hospital, Ferrara, Italy; Irene Gagliardi of the Section of Endocrinology and Internal Medicine, Department of Medical Sciences, University of Ferrara, Ferrara, Italy.

Funding Open access funding provided by Università degli Studi di Ferrara within the CRUI-CARE Agreement.

\section{Compliance with ethical standards}

Conflict of interest The author declares no competing interests.

Publisher's note Springer Nature remains neutral with regard to jurisdictional claims in published maps and institutional affiliations.

Open Access This article is licensed under a Creative Commons Attribution 4.0 International License, which permits use, sharing, adaptation, distribution and reproduction in any medium or format, as long as you give appropriate credit to the original author(s) and the source, provide a link to the Creative Commons license, and indicate if changes were made. The images or other third party material in this article are included in the article's Creative Commons license, unless indicated otherwise in a credit line to the material. If material is not included in the article's Creative Commons license and your intended use is not permitted by statutory regulation or exceeds the permitted use, you will need to obtain permission directly from the copyright holder. To view a copy of this license, visit http://creativecommons. org/licenses/by/4.0/.

\section{References}

1. M. Falconi, B. Eriksson, G. Kaltsas, D.K. Bartsch, J. Capdevila, M. Caplin et al. ENETS consensus guidelines update for the management of patients with functional pancreatic neuroendocrine tumors and non-functional pancreatic neuroendocrine tumors. Neuroendocrinology 103(2), 153-171 (2016)

2. M. Pavel, D. O'Toole, F. Costa, J. Capdevila, D. Gross, R. Kianmanesh et al. ENETS consensus guidelines update for the management of distant metastatic disease of intestinal, pancreatic, bronchial neuroendocrine neoplasms (NEN) and NEN of unknown primary site. Neuroendocrinology 103, 172-185 (2016)

3. D.J. Kwekkeboom, E.P. Krenning, R. Lebtahi, P. Komminoth, B. Kos-Kudła, W.W. de Herder et al. ENETS consensus guidelines for the standards of care in neuroendocrine tumors: peptide receptor radionuclide therapy with radiolabeled somatostatin analogs. Neuroendocrinology 90(2), 220-226 (2009)

4. W.T. Zandee, T. Brabander, A. Blažević, B.L.R. Kam, J.J.M. Teunissen, R.A. Feelders et al. Symptomatic and radiological response to 177Lu-DOTATATE for the treatment of functioning pancreatic neuroendocrine tumors. J. Clin. Endocrinol. Metab. 104(4), 1336-1344 (2019)

5. A. Sowa-Staszczak, D. Pach, R. Chrzan, M. Trofimiuk, A. Stefańska, M. Tomaszuk et al. Peptide receptor radionuclide therapy as a potential tool for neoadjuvant therapy in patients with inoperable neuroendocrine tumours (NETs). Eur. J. Nucl. Med Mol. Imaging 38(9), 1669-1674 (2011)

6. E.I. van Vliet, C.H. van Eijck, R.R. de Krijger, E.J. van Nieveen, J. J. Teunissen, B.L. Kam, Neoadjuvant Treatment of Nonfunctioning Pancreatic Neuroendocrine Tumors with [177Lu-DOTA0,Tyr3] Octreotate. J. Nucl. Med. 56, 1647-1653 (2015).

7. M. Van Essen, E.P. Krenning, M. De Jong, R. Valkema, D.J. Kwekkeboom, Peptide receptor radionuclide therapy with radiolabelled somatostatin analogues in patients with somatostatin receptor positive tumours. Acta Oncologica 46(6), 723-734 (2007)

8. A. Sowa-Staszczak, A. Hubalewska-Dydejczyk, M. Tomaszuk, PRRT as neoadjuvant treatment in NET. Recent Results Cancer Res 194, 479-485 (2013)

9. M. Schiavo Lena, S. Partelli, P. Castelli, V. Andreasi, C.E. Smart, E. Pisa et al. Histopathological and Immunophenotypic Changes of Pancreatic Neuroendocrine Tumors after Neoadjuvant Peptide Receptor Radionuclide Therapy (PRRT). Endocr. Pathol. 31(2), 119-131 (2020)

10. A. Kolasińska-Ćwikła, A. Łowczak, K.M. Maciejkiewicz, J.B. Ćwikła, Peptide receptor radionuclide therapy for advanced gastroenteropancreatic neuroendocrine tumors-from oncology perspective. Nucl. Med. Rev. 21(2), 115-124 (2018).

11. S. Partelli, E. Bertani, M. Bartolomei, C. Perali, F. Muffatti, C.M. Grana et al. Peptide receptor radionuclide therapy as neoadjuvant therapy for resectable or potentially resectable pancreatic neuroendocrine neoplasms. Surgery 163(4), 761-767 (2018)

12. C. Chiapponi, N. Lürssen, B. Cremer, R. Wahba, U. Drebber, M. Faust, Peptide receptor radionuclide therapy as a two-step strategy for initially unresectable liver disease from neuroendocrine tumors: a single-center experience. Endocrine 70(1), 187-193 (2020)

13. T.N. da Silva, M.L.F van Velthuysen, C.H.J van Eijck, J.J. Teunissen, J. Hofland, W.W. de Herder. Successful neoadjuvant peptide receptor radionuclide therapy for an inoperable pancreatic neuroendocrine tumour. Endocrinol. Diabetes Metab. Case Rep. 2018, 18-0015 (2018).

14. S. Ezziddin, H. Lauschke, M. Schaefers, C. Meyer, M. van Essen, H.J. Biersack et al. Neoadjuvant downsizing by internal radiation: a case for preoperative peptide receptor radionuclide therapy in patients with pancreatic neuroendocrine tumors. Clin. Nucl. Med. 37(1), 102-104 (2012)

15. D. Kaemmerer, V. Prasad, W. Daffner, D. Hörsch, G. Klöppel, M. Hommann et al. Neoadjuvant peptide receptor radionuclide therapy for an inoperable neuroendocrine pancreatic tumor. World J. Gastroenterol. 15(46), 5867-5870 (2009)

16. F. Muffatti, S. Partelli, V. Andreasi, A. Piccioli, E. Bertani, M. Bartolomei et al. Outcome of surgical resection after neoadjuvant peptide receptor radionuclide therapy (PRRT) for pancreatic neuroendocrine neoplasms: a case-matched analysis. Pancreatology 17, S83 (2017) 